\title{
Lo impensado de la no-filosofía: Merleau-Ponty 1961-2010*
}

\author{
Luis Álvarez Falcón ${ }^{* *}$ \\ Universidad de Zaragoza
}

Recibido: 2 de octubre de 2009 - Aprobado: 5 de noviembre de 2009

La philosophie a son ombre portée qui n'est pas simple absence de fait de la future lumière.

Maurice Merleau-Ponty

\section{Resumen}

El presente artículo responde al planteamiento inicial de una larga investigación que tuvo su inicio en el año 2008, en el contexto del Coloquio Internacional Merleau-Ponty Viviente (Morelia, Michoacán, México) y del Coloquio Internacional Merleau-Ponty 1908-2008 (Zaragoza, España) y que finalizará en los actos de conmemoración del quincuagésimo aniversario de la desaparición del pensador francés. A lo largo de tres capítulos, veremos desarrolladas las nociones críticas de «no-filosofía» y de lo «impensado», poniendo en relación el pensamiento tardío de Edmund Husserl y las últimas consideraciones de Maurice Merleau-Ponty. Su conclusión nos mostrará la convergencia de ambos pensadores, describiendo la tradición fenomenológica que Merleau-Ponty recogió y las potentes intuiciones que supo anunciar en aquello que definiremos como «lo impensado de su no-filosofía». El resultado final de esta investigación será expuesto en el futuro Coloquio Internacional Merleau-Ponty 1961-2011.

Palabras clave: fenomenología, no-filosofía, lo impensado, pensamiento vertical, lo invisible, epoché, conciencia, subjetividad, ser salvaje.

\footnotetext{
Artículo inscrito en la investigación del Coloquio Internacional Merleau-Ponty Viviente, celebrado en la ciudad de Morelia (Michoacán, México) en el año 2008.

** Doctor en Filosofía de la Universidad de Valladolid y profesor de Filosofía de la Universidad de Zaragoza. Se ha destacado por sus numerosos estudios en los ámbitos de la Ontología, de la Teoría del Conocimiento y de la Estética. Ha dirigido y coordinado la conmemoración en España del aniversario del nacimiento de Mauricie Merleau Ponty y es miembro asociado del Círculo Latinoamericano de Fenomenología (CLAFEN). Miembro fundador de la cátedra internacional Merleau-Ponty, celebrada en la ciudad de Morelia (México) en el 2008. Correo electrónico: falcon@unisar.es
} 


\section{The unthought-of in non-philosophy: Merleau-Ponty 1961-2011}

\section{A bstract}

The present article emerges from the initial positions of the extensive investigation that sprung in 2008 from the context of the Coloquio Internacional Merleau-Ponty Viviente -Morelia, Michoacán, Mexico- and from the International Conference Merleau-Ponty 1908-2008 -Zaragoza, Spain- and that will conclude with the commemoration of the fiftieth anniversary of the French thinker's death. Along three chapters, we will develop the critical notions of «non-philosophy» and of "the unthought-of» by relating the later thoughts of Edmund Husserl and Maurice Merleau-Ponty's last considerations. Our conclusions will prove the convergence of both thinkers when describing the phenomenological tradition that Merleau-Ponty inherited and the influential intuitions that he announced in what we will define as «the unthought-of of his non-philosophy». The final result of this investigation will be exposed in the upcoming International Conference Merleau-Ponty 1961-2011.

Key words: Phenomenology, Non-philosophy, The unthought-of, Vertical thought, The invisible, Epoché, Conscience, Subjectivity, Wild Being. 


\section{La non-pensée de la no- philosophie. Merleau-Ponty 1961- 2010}

\section{Résumé}

Cet article répond au problème initial posé au cours d'une longue recherche qui eut son début en 2008 dans le cadre du colloque international Merleau - Ponty Viviente, Morelia, Michoacán, Mexico et du Colloque internacional Merleau - Ponty 1908- 2008 , Zaragoza Espagne et qui prend fin pendant les actes de commémoration du cinquantième anniversaire de la disparution du penseur francais. Nous verrons, au cours de trois chapitres, le dévelopement des notions critiques de notre "philosophie" et de "la non- pensée", tout en mettant en relation la pensée retardée de Edmunsd Hussurl" et les dernières considérations de Maurice Merleau-Ponty. Sa conclusión nous montrera la convergence des dueux penseurs . Cette conclusión décrit la tradition phénomélogique que Merleau - Ponty a recueilli et les puisantes intuitions qu'il a pu annoncer ce qu La non- pensée de la no- philosophie

Mots clés: phenomenology, no-philosophie, impensé, pensée vertical, Invisible, époché, la conscience, la subjectivité, être sauvage 


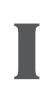

Entre los años 1960 y 1961, justo antes de su repentina desaparición, en los cursos Husserl en los límites de la fenomenología' (Merleau-Ponty, 1998, pp. 11-92) y Filosofía y no-filosofía después de Hegel2 (Merleau-Ponty, 1996, p. 163), Maurice Merleau-Ponty expondrá su concepción personal sobre el pensamiento de Husserl. Dos textos tardíos, pero fundamentales en la etapa final de Husserl, pondrán de relieve la extraña concordancia de los intereses de ambos en los últimos años de sus vidas: El origen de la Geometría, anexo III de la Krisis (Husserl, 1969) del año 1932, publicado por Eugen Fink en 1939, y un texto de 1934, publicado por Martin Farber en 1940, Inversión de la doctrina copernicana en la interpretación de la visión habitual del mundo. El arjé-originario Tierra no se mueve. Investigaciones fundamentales sobre el origen fenomenológico de la corporeidad, de la espacialidad de la naturaleza (Husserl, 1940, pp. 307-325). Merleau-Ponty mostrará un especial interés por estos dos trabajos, y el curso de $1960^{3}$ girará en torno a las potentes intuiciones que Husserl expondrá en ellos (Merleau-Ponty, 1968, pp. 169-173).

Tan sólo un año antes, coincidiendo con el centenario del nacimiento de Husserl, y mientras Eugen Fink presentaba su importante aportación "La filosofía tardía de Husserl en la época de Friburgo", en el No. 4 de Phaenomenologica, bajo la dirección de Van Breda y Taminiaux, nuestro autor publicará su artículo "El filósofo y su sombra"4 (Merleau-Ponty, 1960, pp. 195-220). En sus líneas se advertirá la efectividad propia de la fenomenología y de sus propias y primitivas intenciones, recordando el planteamiento inicial que ya aparecía en Ideen II:

1 Curso del lunes, en el Collège de France, enero-mayo de 1960. Notas de preparación de Merleau-Ponty, Biblioteca Nacional, volumen XVIII, 51 ff.; Transcripción, presentación y anotaciones de Franck Robert, en Notes de cours sur L'origine de la géométrie de Husserl, seguido de Recherches sur la phénoménologie de Merleau-Ponty, bajo la dirección de Renaud Barbaras.

2 Curso del lunes, en el Collège de France, enero-mayo de 196. Notas de preparación de Merleau-Ponty, Biblioteca Nacional, volumen XX, 145 ff.; texto presentado por Claude Lefort en Textures, No. 8-9, 1974, pp. 83-129 y No. 10-11, 1975, pp. 145-173, recogido en Notes de cours 1959-1961.

3 Resumen del curso del lunes, en el Collège de France. Annuaire du Collège de France, 60 pp. 169-173; recogido en Résumés de cours 1952-1960.

4 También se encuentra en Van Breda, H.-L. \& Taminiaux, J. (ed.). (1959). Edmund Husserl 1859-1959. Phaenomenologica, (4), 195-220. 
Quand on dit que la chose perçue est saisie "en personne" ou "dans sa chair" (leibhaft), cela est à prendre à la lettre: la chair du sensible, ce grain serré qui arrête l'exploration, cet optimum qui la termine reflètent ma propre incarnation et en sont la contrepartie. Il y a là un genre de l'être, un univers avec son "sujet" et son "objet" sans pareils, I'articulation de l'un sur l'autre et la définition une fois pour toutes d'un "irrélatif" de toutes les "relativités" de l'expérience sensible, qui es "fondement de droit" pour toutes les constructions de la connaissance (Merleau-Ponty, 1960, p. 272).

Este "irrelativo" de todas las "relatividades" abrirá el paso a la efectividad de los niveles de experiencia en su dimensión "vertical", a un pensamiento comprometido con la experiencia antepredicativa, que exige recuperar y prolongar un saber anónimo y pre-categorial (Bech, J., 2005, p. 61). Se tratará en definitiva de un modelo de pensamiento que, tal como definirá Merleau-Ponty en el curso del lunes de 1961, operará en lo imaginario, o más bien en la Phantasia, puesto que se considerará a sí mismo como su expresión y, por consiguiente, jamás se separará de él. Cuando el pensamiento interrogue radicalmente de esta forma hará posible una «filosofía de la no-filosofía».

En efecto, en 1961, a escasos días de su desaparición, Merleau-Ponty hablará de la filosofía como de una «no-filosofía» (Merleau-Ponty, 1996, pp. 269-352). Ya en 1959, en su mencionado artículo "Le philosophe et son ombre", nuestro autor advertía que Husserl había planteado esta cuestión en la exploración de la arquitectónica que configura los diferentes niveles de la experiencia, capas escalonadas (couches étagées), y cuya estructura no puede ser de por sí eidética, puesto que la propia filosofía aparece como un modo de institucionalización simbólica en uno de esos niveles. Entre las "capas profundas" y las "capas superiores" de la constitución situará esa singular relación de Selbstvergessenheit (olvido de sí mismo), tematizada por Schopenhauer ${ }^{5}$, que ya Husserl había avistado en Ideen II, y que MerleauPonty situará, siguiendo a Kant, en un «Logos du monde esthétique» (Merleau Ponty, 1960, pp. 281-282).

5 " [...] un estado de pura contemplación, de apertura a la intuición, que nos lleva a perdernos en el objeto y a olvidarnos de cualquier individualidad, superando el conocimiento regulado por el principio de razón suficiente", en Schopenhauer (2004, p. 150). 
Las vivencias de la conciencia estarán siempre, explícita o implícitamente, aprehendidas en el tejido extremadamente complejo de un doble encadenamiento: "horizontal", conforme a lo que constituye la coherencia (racional) de la vida de la conciencia; y "vertical", según el cual no habrá vivencia que no implique una base y un fundamento (Fundament) sobre el que se edifique toda la profundidad y estratificación de la experiencia (Álvarez Falcón, 2009). La filosofía, como institución simbólica racional, en su dimensión meramente horizontal, se habrá vuelto impositiva. Será un pensamiento de survol, una instancia de sobrevuelo que impone el sentido, ya sea desde un supuesto Ser en el límite superior, desde unas condiciones trascendentales de posibilidad, o desde un límite inferior, desde los datos como realidades últimas.

Tal "filosofía" vivirá sobre todo en el pasado, fósil y estancada como una historia de la filosofía. La no-filosofía será, en palabras de Merleau-Ponty en la preparación del curso del 6 de marzo de 1961, "la filosofía verdadera» (Merleau-Ponty, 1996, p. 312), es decir, una filosofía de la experiencia que se da entre las diferentes couches étagées (estratos escalonados), intencionalidades que no pueden adecuarse a un espíritu constituyente universal y cuya articulación es no-eidética. Esta no-filosofía será únicamente posible, o bien como "luz negra" de la fenomenología, capaz de exhibir todo lo que queda oculto y encubierto por la antesala del eidos, y que parece quedar fuera de todo orden simbólico, o bien como el hermano bastardo del inmenso y razonado desarreglo (dérèglement) de todos los sentidos: el Arte. Se tratará, pues, de una filosofía negativa que deberá entenderse, tal como posteriormente insinuará el propio Jan Patoĉka en sus ya célebres ensayos ${ }^{6}$, como una «a-filosofía». En esto, Merleau-Ponty será concluyente al recordar en el citado curso del lunes, en el Collège de France, este locus classicus o fragmento clave:

Il s'agit d'une philosophie qui veut être philosophie en étant non-philosophie, d'une «philosophie négative» (au sens de «théologie négative»), qui s'ouvre accès à l'absolu, non comme «au-delà», second ordre positif, mais comme un

6 Véase Patoĉka, J. "El subjetivismo de la fenomenología husserliana y la posibilidad de una fenomenología asubjetiva" y "El subjetivismo de la fenomenología husserliana y la exigencia de una fenomenología asubjetiva". 
autre ordre qui exige l'en-deçà, le double, n'est accessible qu'à travers lui-la vraie philosophie se moque de la philosophie, est a-philosophie (MerleauPonty, M. o. c., p. 275).

El interés de nuestro autor por esta «a-filosofía» nos anticipará el giro inesperado que la fenomenología dará a partir del año 1966, con la aparición en la edición de la Husserliana de las investigaciones sobre la Síntesis Pasiva (Husserl, 1966), y de otros textos como la Fenomenología de la Intersubjetividad (Husserl, 1973), los pasajes de las lecciones de 1907 sobre Cosa y Espacio (Husserl 1973), editadas también en el año 1973, o Phantasia, conciencia de imagen y recuerdo (Husserl 1980), y un largo etcétera (Álvarez Falcón, 2009). Si bien Merleau-Ponty no podrá conocer la mayoría de estas ediciones, habrá sido capaz, sin embargo, de intuir el despliegue implícito en el pensamiento de Husserl: el recorrido "vertical", de ida y vuelta, entre las regiones de lo Visible y de lo Invisible. El sentido auténtico de la fenomenología, tal como nos describirá Renaud Barbaras en su ensayo sobre la ontología de MerleauPonty (Barbaras, 2001), será la tentativa de llevar la experiencia muda a la expresión pura de su propio sentido, desarrollando una parte de no-filosofía, un orden que se resiste a la conciencia constituyente.

Aunque el término «no-filosofía» (Merleau-Ponty, 1997 y 2001), acuñado como tal, no apareciese más que discretamente mencionado en sus últimos cursos (Saint-Aubert, 2006, p. 61 y ss.), la extensión y radicalidad de su significado teórico comenzará a exhibirse desde el inicio de sus planteamientos fenomenológicos. De este modo, la verdad de la fenomenología residirá en el lugar mismo de sus límites, en el confín mismo de su "sombra", en donde comienza su relación con la «a-filosofía», o con la «no-filosofía». Esta"sombra" hará referencia a tres umbrales muy significativos, y que Merleau-Ponty habrá puesto al descubierto, llevando al límite el programa de la fenomenología de Husserl, en cuanto filosofía de la experiencia que se da necesariamente entre estratos resonantes. En primer lugar, tal "sombra" hará referencia al eco persistente de ambos pensadores tras el cruce teórico de sus herencias inconclusas, en el advenimiento de una nueva concepción fenomenológica de la filosofía. En segundo lugar, su fulgente oscuridad nos traerá la noticia del umbral de acceso a la «no-filosofía» en los límites de un ascenso vertical de la reducción. En tercer lugar, este subumbrare será el testimonio de la zona 
o región a la que, por una u otra causa, no llega la actividad constituyente del "yo", delimitando la patencia de un límite, de un final y de un comienzo, de un origen que procede de la cesación de las luces y que tiene su comienzo en una nueva penumbra: un en-deça y un au-delà.

Si tras el giro copernicano, el pensamiento de Kant determinó la primera inversión trascendental, la fenomenología, como "luz oscura" de la nofilosofía, habrá dispuesto con la máxima claridad esta segunda inversión: la inversión entre el Ser y el Fenómeno. Mientras el Ser se refugiará en la estabilidad de lo Visible, la no-filosofía producirá un descentramiento que irá desde la identidad y la posicionalidad a la pluralidad y la indeterminación del Fenómeno. Merleau-Ponty será preciso al advertir que «La realidad es un tejido sólido» (Merleau-Ponty, 2000). Lo que hay, eso que es lo Invisible, plural, no figurado e indeterminado, es la riqueza de la realidad que se va empobreciendo al contraerse por centramiento al territorio de lo Visible. La no-coincidencia y el inevitable sobreadvenimiento de este horizonte soberano e inaccesible dará primacía a la novedad y la imprevisibilidad de aquello que el propio Heidegger calificara de Impensado (das Ungedachte), o"sombra" que acompaña a todo pensamiento impositivo, a toda filosofía de la conciencia que desde el pasado sobrevuela como instancia de survol, imponiendo el sentido, en una Sinngebung capaz de dominar el mundo en vez de verse hundida en él.

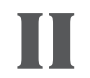

En el citado curso de los lunes de 1961 (Merleau-Ponty, 1974, pp. 269-352), Merleau-Ponty utilizará el ejemplo de un pensamiento que, a pesar de presentarse como filosofía, resultará ser un paradigma de la no-filosofía. Nos referimos a la introducción de doce páginas en dieciséis párrafos que Hegel añadió a su Fenomenología del Espíritu. El fenómeno como Erscheinung (Aparición), y no como Darstellung (lo que aparece), emergerá como relación con el absoluto, pero no como Ser sino como schon bei uns, como algo que ya está previamente entre nosotros y que ha de desvelarse por su propio esplendor. Tal texto ya había sido comentado por Martin Heidegger en su seminario de 1943, pero en este caso será esgrimido por Merleau-Ponty para anunciar 
esa segunda inversión, más allá de la inversión trascendental kantiana, que se ha producido en la fenomenología.

En la página 202 de Signes, en la edición original de 1960, dentro de su modesta contribución al centenario del nacimiento de Husserl, nuestro autor expondrá centralmente esta concepción de lo impensado, que desde su origen y lamento heideggeriano dejará importantes secuelas en toda su obra. De este modo, aquí podremos advertir, además de la presencia fulgurante de los versos de Angelus Silesius, aquella cita de la lección novena de La proposición del fundamento en torno a Kant y Leibniz:

Cuanto más grande es la obra del pensar de un pensador -cosa que en absoluto queda cubierta por la extensión y el número de sus escritos- tanto más rico es lo impensado que hay en esta obra del pensar, es decir, aquello que por primera vez y únicamente por esta obra del pensar aflora como lo aún no pensado. Esto no pensado no atañe desde luego a algo que un pensador haya pasado por alto o sido incapaz de dominar, y que después una posteridad más sabia tuviera que retomar (Heidegger, 2003, p. 106).

Lo propio de un pensamiento es lo que busca todavía por decir, su Impensado, que solamente puede revelarse en una reflexión, convirtiéndose en el eco de aquella región que está en-deça u au-delà de la filosofía misma como Institución simbólica racional (Stiftung). En este sentido, en la confrontación con Husserl que Merleau-Ponty llevará a cabo será difícil separar lo que pertenece a cada uno de ellos; más bien, será preciso advertir que las tesis del último Husserl y las tesis del último Merleau-Ponty terminarán confluyendo, precisamente, en aquello que por primera vez, y únicamente por esta obra del pensar, aflora como lo aún no pensado: su no-filosofía.

A la edición póstuma del curso sobre Philosophie et non-philosophie depuis Hegel habrá que añadir una edición más reciente, pero también fundamental, que llevará por título: La philosophie aujourd 'hui (Merleau-Ponty, 1996). Este será el curso, en principio sin título, que el autor impartirá en el Colegio de Francia, entre enero y mayo de 1959. En este curso, Merleau-Ponty se aproximará, quizá más que nunca, a la "sombra" inconclusa del maestro, distinguiendo la articulación precisa de su pensamiento en torno a tres ejes 
principales. Por un lado, el eje de los descubrimientos en torno a la relación «intencionalidad, esencia y facticidad»; por otro lado, la regresión idealista de la fenomenología; y por último, la retractación del idealismo y la profundización en el pensamiento vertical. Estos tres ejes corresponderán a su vez con el periodo inicial de Investigaciones Lógicas; con el periodo idealista, y más ortodoxo, de Ideas relativas a una fenomenología pura y una filosofía fenomenológica; y por último, con el periodo de Meditaciones Cartesianas. En este último intervalo, la investigación sobre las dimensiones verticales aproximará al autor a las nociones de Leib, pasividad e intersubjetividad, que serán, en definitiva, las nociones a partir de las cuales Merleau-Ponty desarrollará toda su concepción de la fenomenología en su aproximación a lo Impensado por ambos pensadores. La caracterización de una «pasividad originaria» pondrá en tela de juicio la concepción de la reflexión, o reducción trascendental, desvelando esa estratificación escalonada y vertical en la que aparecerá lo no tético en la naturaleza misma de la conciencia y contra la adecuación misma de un espíritu constituyente universal (Merleau-Ponty, 1996, p. 68).

Desde el curso de 1959, y teniendo en cuenta los citados cursos de 1960 y 1961 («Husserl en los límites de la fenomenología» y «Filosofía y no-filosofía después de Hegel»), Merleau-Ponty intentará incansablemente pensar el régimen arquitectónico de un pensamiento vertical, repensando a su vez la noción misma de pasividad frente a la actividad constituyente del "yo". Desde la región de lo Visible, desde las nociones de sujeto, naturaleza y síntesis activa, ascenderá, o descenderá, a la región de lo Invisible, en donde descubrirá el Leib, la hylé y la proto-hylé, y las síntesis pasivas; en definitiva, sus controvertidas nociones de la chair, del quiasmo, y de las wesen sauvages. La fenomenología se habrá convertido en ese recorrido de ida y de vuelta entre lo Visible y lo Invisible. Merleau-Ponty estará más cerca que nunca de eso que había de Impensado en la obra del pensar husserliano. De ahí que en las Notes de travail de su inconclusa obra Le visible et l'invisible, en noviembre del mismo año 1959, confirma que la filosofía no había hablado jamás de la pasividad de nuestra actividad, es decir, citando a Valéry, de un «cuerpo del espíritu» (corps de l'esprit). Y aquí, me permitiré la licencia de reproducir en toda su extensión la claridad de esta pequeña anotación: 
L'âme pensé toujours: c'est en elle une propriété d'état, elle ne peut pas ne pas penser parce qu'un champ a été ouvert où s'inscrit toujours quelque chose ou l'absence de quelque chose. Ce n'est pas là une activité de l'âme, ni une production de pensées au pluriel, et je ne suis pas même l'auteur de ce creux qui se fait en moi par le passage du présent à la rétention, ce n'est pas moi qui me fais penser pas plus que ce n'est moi qui fais battre mon cœur. Sortir par là de la philosophie des Erlebnisse et passer à la philosophie de notre Urstiftung... (Merleau-Ponty, 1999, p. 270).

La conclusión será radical, rotunda y definitiva: salir de la filosofía de las vivencias (Erlebnisse) y pasar a la filosofía de nuestra institución originaria de sentido (Urstiftung), es decir, de nuestra protofundación o protoinstitución. En definitiva, su propuesta anunciará la necesidad de prescindir de un pensamiento "horizontal", de una filosofía de la conciencia, aplastantemente configurada en un pensamiento de survol, y ascender "verticalmente" en busca de la estratificación originaria en dónde resituar la «no-filosofía» de lo impensado, tanto por Husserl como por él mismo y por toda la tradición de una filosofía cuyo origen, exigencia y necesidad sólo puede presentarse como filosofía fenomenológica. Tal radical pretensión pasará por aquel dérèglement que el mismo Rimbaud ejemplificase, anunciado por esa extraña y providente misiva merleau-pontiana que tantas resonancias trae al pensamiento contemporáneo: «no soy yo quien me hace pensar como no soy yo quien hace latir mi corazón».

El mismo Husserl, en sus últimos trabajos, y contra el supuesto idealista que había sostenido en el periodo de Ideas, apoyará este combate contra la filosofía de la conciencia. El descubrimiento de la subjetividad como Leib quinestésico, la transposibilidad de las «síntesis pasivas» frente a la posibilidad de las síntesis activas, y el horizonte ineludible de la «interfacticidad» frente a la intersubjetividad de la identidad simbólica, terminarán por distinguir un pensamiento vertical en su propia genealogía de la verdad, y la filosofía de Merleau-Ponty acabará por convertirse en «no-filosofía», cuyo objeto de estudio será, en definitiva, el fenómeno en cuanto fenómeno, es decir, ese "ser salvaje" (être sauvage), vertical y ontológicamente primero, que ocupa el dominio mismo de lo Invisible en el límite más extremo de la epoché. 


\section{III}

Por extraño que pueda parecer, lo Impensado de la no-filosofía de Maurice Merleau-Ponty aparecerá ya anunciado en 1945, en la nota 9 del capítulo IV de la primera parte de su Phénoménologie de la perception. Recordemos que este capítulo estaba dedicado a "La síntesis del propio cuerpo" y exponía las cuestiones de la espacialidad y la corporeidad, de la unidad del cuerpo y de la obra de arte y del hábito perceptivo como adquisición de un mundo. Pues bien, al tratar precisamente del hábito y utilizando el ejemplo del "bastón del ciego", Merleau-Ponty hará una certera alusión a Husserl:

Husserl, por ejemplo, definió durante largo tiempo la conciencia o la imposición de un sentido por el esquema Auffassung-Inhalt y como una beseelende Auffassung. Un paso definitivo lo da al reconocer, desde las Conferencias sobre el tiempo, que esta operación presupone otra más profunda por la que el contenido se prepara para esta captación. "Toda constitución no se hace según el esquema Auffassunginhalt-Auffassung" (Merleau-Ponty, 2000, p. 169).

En efecto, en Las Lecciones sobre la conciencia interna del tiempo del año 1905, en su Introducción, y al hablar de la "Desconexión del tiempo objetivo», en concreto, al exponer la distinción entre un tiempo «sentido» y un tiempo percibido, Husserl hace la siguiente advertencia:

"Lo sentido" indicaría, pues, un concepto de relación que por sí solo nada diría acerca de si lo sentido es sensual, siquiera acerca de si es inmanente en el sentido en que lo sensual lo es. Quedaría abierto, en otras palabras, si lo sentido mismo está ya constituido, y quizá de un modo muy distinto del de lo sensual. Pero toda esta diferenciación es mejor dejarla al margen. No toda constitución responde al esquema "contenido de aprehensión-aprehensión" (Husserl, 2002, p. 29).

En estos dos textos convergerá lo Impensado de ambos pensadores, con la expresa advertencia de que esta cuestión, que según Husserl, y en ese momento, "es mejor dejarla al margen", conllevará tanto todo el despliegue del pensamiento merleau-pontiano como el despliegue mismo que el pen- 
samiento husserliano mostrará en las mencionadas ediciones posteriores a 1966 y que Merleau-Ponty nunca llegará a conocer. Valga advertir que Rudolf Boehm, el editor de Sobre la fenomenología de la conciencia temporal inmanente (1893-1917), ya hará una especial referencia a esta nota en el tomo X de la Husserliana (Husserl, 1969). Parece, pues, que no es posible generalizar la intencionalidad entendida como imposición de sentido. Esto sería contradictorio con la noción de fenómeno en tanto modo de aparición (Erscheinung). Por consiguiente, la conciencia es algo más que actividad, y ese algo más no es consistente con el esquema Auffassunginhalt-Auffassung (Contenido de aprehensión-Aprehensión).

En efecto, encontraremos una pasividad originaria en el interior mismo de la conciencia, y aquí Merleau-Ponty desarrollará aquella intuición que ya había anunciado en el Prólogo de su Fenomenología de la percepción (Merleau-Ponty, 2000, pp. 17-18), al advertir, tras citar la Crítica del juicio, que Husserl ya había distinguido la intencionalidad de acto de otro tipo de intencionalidad, una noción más ampliada de intencionalidad que permitiría a la fenomenología convertirse en una fenomenología de la génesis y al pensamiento en un pensamiento vertical de los diferentes estratos, o capas escalonadas (couches étagées), anteriores a los actos. Se trataba de la intencionalidad operante y latente (fungierende Intentionalität) que constituiría la unidad natural y antepredicativa del mundo y de nuestra vida, la que se manifestaba en nuestros deseos, nuestras evaluaciones, nuestro paisaje, de una manera más clara y rotunda que en el conocimiento objetivo.

En los doce años que transcurren entre las dos ediciones de Investigaciones Lógicas, la de 1901 y la de 1913, el pensamiento de Husserl sufrirá una crisis profunda y una honda transformación. Se hará patente lo que hasta entonces había permanecido de un modo latente y casi oculto: los resortes filosóficos básicos de la reducción y la constitución. Esta crisis aparecerá ostensiblemente en el citado curso de 1905 sobre la conciencia íntima del tiempo, que editará Heidegger en 1928, y en el curso de 1907 sobre la idea de fenomenología, que no aparecerá editado hasta 1950, por Biemel, en el volumen II de la Husserliana (Husserl, 1973). Las modificaciones introducidas en la segunda edición de las Investigaciones, tal como se puede apreciar en la edición definitiva de 
Elmar Holenstein, en 1975, en el volumen XVIII y siguientes de la Husserliana (Husserl, 1975), son un testimonio de esta autoconciencia operada, que en 1901 es todavía una intuición no consciente de sus consecuencias. La crisis de 1905 y la primera exposición formal de la fenomenología del curso de 1907 serán el fiel testimonio de la conexión entre la primera edición de las Investigaciones de 1901 y la segunda de 1913. En este fecundo periodo, entre mayo y agosto de 1907, en Gotinga, Husserl impartirá la segunda parte de un curso titulado inicialmente: «Fragmentos principales de la fenomenología y de la crítica de la razón». Tal curso corresponderá al texto del manuscrito husserliano Fl13, llamado por Husserl «Dingvorlesung».

Las cinco lecciones de introducción general a la fenomenología transcendental de dicho curso serán, tal como hemos apuntado, publicadas por Biemel en 1950, bajo el título: Die Idee der Phänomenologie. Sin embargo, el gran grueso del curso no será publicado hasta 1973, cuando Ulrich Claesges, en el volumen XVI de la Husserliana, edite estas lecciones, bajo el enigmático título: Ding und Raum, Vorlesungen 1907. El traductor de la edición francesa, Jean-François Lavigne, será concluyente en su introducción al confirmar un hecho filosóficamente decisivo en la historia del pensamiento contemporáneo: las lecciones del verano de 1907 sobre la cosa espacial inaugurarán la fenomenología de la percepción (Husserl, 1989, p. 5). Es evidente que Merleau-Ponty no podrá conocer este texto, pero lo sorprendente resultará al comprobar que, muy a pesar de ello, habrá sido consciente de la trascendencia teórica que conllevan sus conclusiones.

En la página 285 de la edición de Ulrich Claesges, en correspondencia con la lección final del 3 de agosto de 1907, Husserl comenzará de este modo su última consideración:

Pertenece a la esencia de la cosa en general ser una unidad intencional idéntica que se "constituye" en una cierta multiplicidad de apariciones efectivas o posibles, se legitima según su ser y su ser-así respectivo en el encadenamiento de apariciones reglado y cada vez motivado. Pero el encadenamiento es un encadenamiento de apariciones entre-acordadas, que se llenan unas en otras, y son portadas por una conciencia de creencia que las atraviesa, o, si se prefiere, una conciencia posicional, conciencia de ser. Saber qué relación mantiene esta 
conciencia posicional con las simples apariciones necesitaría de investigaciones más profundas (Husserl, 1973, p. 285).

La relación imposible entre esta consideración y la mencionada nota No. 9 del capítulo IV de la primera parte de la Fenomenología de la Percepción, nos hará suponer que la convergencia de ambos pensadores tiene lugar en la sombra misma de sus Impensados, a través, en el caso de Merleau-Ponty, del conocimiento previo de Las Lecciones sobre la conciencia interna del tiempo del año 1905, en donde Husserl ya advierte esta propuesta radical (Husserl, 2001). Otra cosa sería ya elucubrar sobre el derrotero que el pensamiento merleau-pontiano hubiera tomado si en 1961 no se hubiera cumplido el fatal designio de Rimbaud: «on me pensé».

Rota la estructura bimembre Auffassunginhalt-Auffassung (Contenido de aprehensión- Aprehensión), la "vertical" quedará abierta para un ascenso hacia lo Impensado, es decir, para aquella no-filosofía que tanto Husserl como Merleau-Ponty vislumbraron como la extraña distancia (écart) que separa al sujeto mismo y que aleja cada cosa de su posible identidad (Bech. J. M., 2003, pp. 57-94). Ambos establecerán una nueva estructura trimembre: AuffassungErscheinung-Darstellung (Aprehensión o acto intencional, Aparición o contenido de aprehensión, Exposición u objeto). De este modo, Merleau-Ponty estaba en lo cierto al advertir que la conciencia o la imposición de sentido seguía el esquema husserliano Auffassung-Inhalt, y beseelende Auffassung (Aprehensión animada), y que, por consiguiente, suponía otra operación más profunda mediante la cual el contenido era preparado para la aprehensión. Todo el pensamiento merleau-pontiano partirá de esta consideración.

La estructura bimembre de la percepción será la filosofía de la conciencia que Merleau-Ponty descalificará por su naturaleza impositiva y de "sobrevuelo"; aquella que anula el eje vertical de la Erscheinung, de la Aparición. Entre la intencionalidad de la Auffassung, de la Aprehensión o el Acto, y la identidad de la Darstellung, Exposición u Objeto, habrá una extraña connivencia, una complicidad que abrirá la vertical de las diferentes couches étagées (estratos escalonados) sobre el eje de la Erscheinung, de la Aparición. Aunque tanto la Erscheinung (Aparición) como la Darstellung (Exposición) sean ambas "fenómenos", el eje de la Erscheinung (Aparición) será el eje de los fenómenos 
en sentido estricto: el fenómeno en cuanto fenómeno. Esta vertical nos pondrá directamente en contacto con la trascendencia, con el "ser salvaje" (être sauvage), vertical y ontológicamente primero.

Como es bien sabido (Husserl, 2002), Husserl doblará la reducción trascendental mediante una reducción eidética, es decir, que en su etapa idealista (Ideen) utilizará la «reducción trascendental» (reducción cartesiana, kantiana y psicológica) para partir de la Auffassung (Aprehensión) y llegar a una "Subjetividad trascendental", y, a su vez, tal reducción arrastrará una «reducción eidética» para partir de la Darstellung (Exposición) y llegar a las primeras síntesis. Sin embargo, Merleau-Ponty propondrá una reducción a partir del fenómeno en tanto fenómeno, de la Erscheinung (Aparición). Tal reducción por la vertical central será completamente autónoma e irá desde la Erscheinung, o Apariencia, a la Ereignis, o Quiasmo. Este será el eje que propiciará una «no-filosofía» frente a una filosofía de survol que sobrevuela e impera, es decir, la vía de acceso hacia lo Invisible, hacia lo Impensado de la «no-filosofía». Merleau-Ponty se enfrentará al Husserl idealista a favor del pensamiento último del maestro, a pesar de no haber llegado a vislumbrar más que su "sombra" inconclusa, sustituyendo la «reducción trascendental» y la «reducción eidética» por una verdadera «reducción central fenomenológica", accediendo de este modo al verdadero espesor del mundo, de la naturaleza, de la realidad en definitiva.

Aunque esta estructura trimembre (Anexo) no se mantenga en su ascenso desde lo Visible, por el pensamiento vertical, hacia la región de lo Invisible, su controvertido concepto de la Chair se corresponderá en la reducción con el sujeto y los actos de aprehensión intencional; los esquicios o contenidos de aprehensión se corresponderán a su vez con su noción principal de Quiasmo; y las Eide, esencias o eidos, se corresponderán con las Wesen sauvages. En definitiva, y tal como nos advertirá el propio pensador, encontraremos en el Ser bruto, salvaje, vertical, presente, una dimensión que no es la de la representación ni la del en-sí. El resto de lo Impensado en Maurice Merleau-Ponty se irá ubicando escalonadamente en los diferentes registros gnoseológicos que son, concomitantemente, registros de realidad y que el propio Edmund Husserl, a partir de su "sombra", de las ediciones que irán apareciendo desde el año 1966, configurará en una tectónica de regímenes bien diferenciados, 
en una «no-filosofía» de lo Impensado, del Ser vertical, tal como en adelante describiré, en lo que probablemente serán las Notas de Curso de una futura publicación?

\section{Anexo: Estructura trimembre}

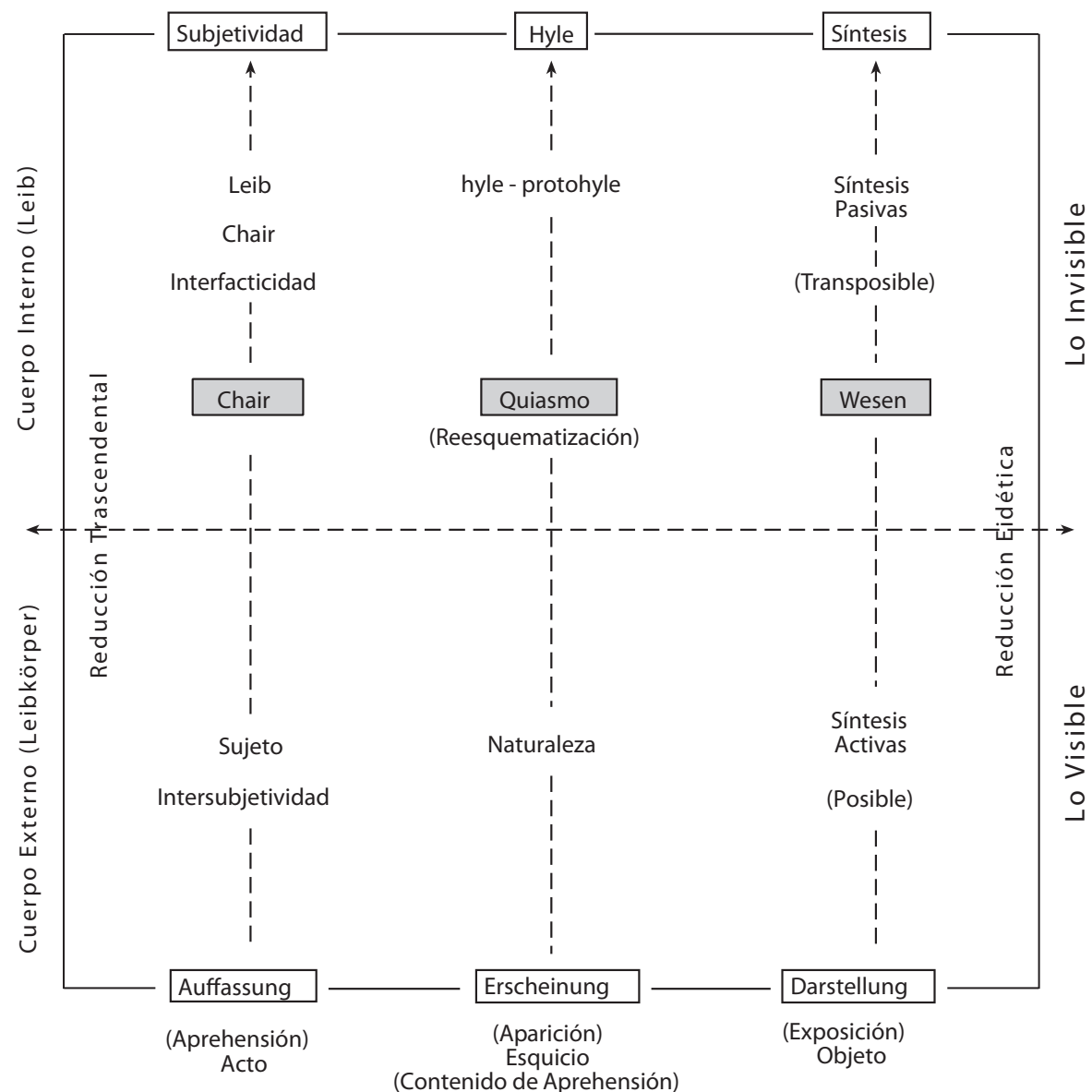

7 Álvarez Falcón, L. Origen, exigencia y necesidad de la fenomenología (Fenomenología, Ontología y Estética). Curso impartido en el otoño de 2009, en el Instituto de Investigaciones Filosóficas "Luis Villoro" de la Universidad Michoacana de San Nicolás de Hidalgo, Morelia, México. 


\section{Referencias}

Álvarez Falcón, L. (2009). Comunidad, historia y sentido. En: IX Congreso Internacional de Fenomenología. España.

Álvarez Falcón, L. (2009). Realidad, arte y conocimiento. La deriva estética tras el pensamiento contemporáneo. Barcelona: Editorial Horsori.

Barbaras, R. (2001). Del'être du phénomène. Surl'ontologie de Merleau-Ponty. Grenoble: Éd. Jérôme Million.

Bech, J. M. (2003). El pensamiento de la no-coincidencia. Revista Convivium. No. 16, 57-94.

Bech, J. M. (2005). Merleau-Ponty: una aproximación a su pensamiento. Barcelona: Anthropos.

Heidegger, M. (2003). La proposición del fundamento. Barcelona: ediciones del Serbal.

Husserl, E. (1940). Umsturz der kopernikanischen Lehre in der gewöhnlichen weltanschaulichen Interpretation. Die Ur-Arche Erde bewegt sich nicht. Grundlegende Untersuchungen zum phänomenologischen Ursprung der Köperlichkeit der Räumlichkeit der Natur in ersten naturwissenschaftlichen Sinne. Alles notwendige Anfangsuntersuchungen. Cambridge: Marvin Farber.

Husserl, E. (1966). Analysen zur passiven Synthesis. Aus Vorlesungs- und Forschungsmanuskripten, 1918-1926. Netherlands: Edited by Margot Fleischer. The Hague, Martinus Nijhoff.

Husserl, E. (1969a). Die Krisis der europäischen Wissenschaften und die transzendentale Phänomenologie, Einleitung in die Phänomenologische Philosophie. La Haya: W. Biemel - Martinus Nijhoff.

Husserl, E. (1969b). Zur Phänomenologie des inneren Zeitbewusstesens (18931917). Netherlands: Rudolf Boehm - Martinus Nijhoff - The Hague. 
Husserl, E. (1973a). Die Idee der Phänomenologie. Fünf Vorlesungen. Netherlands: Walter Biemel - Martinus Nijhoff - The Hague.

Husserl, E. (1973b). Ding und Raum. Netherlands:The Hague - Martinus Nijhoff.

Husserl, E. (1973c). Zur Phänomenologie der Intersubjektivität. Texte aus dem Nachlass. Erster Teil. 1905-1920. Zweiter Teil. 1921-28. Dritter Teil. 192935. Edited by Iso Kern. The Hague, Netherlands: Martinus Nijhoff.

Husserl, E. (1975). Logische Untersuchungen. Netherlands: Elmar Holenstein - Martinus Nijhoff - The Hague.

Husserl, E. (1980). Phäntasie, Bildbewusstsein, Erinnerung. Zur Phänomenologie der anschaulichen Vergegenwartigungen. Texte aus dem Nachlass (18981925). Netherlands: Eduard Marbach - The Hague - Martinus Nijhoff.

Husserl, E. (1984). Logische Untersuchungen. Zweiter Teil. Untersuchungen zur Phänomenologie und Theorie der Erkenntnis. Netherlands: Martinus Nijhoff, The Hague.

Husserl, E. (1989a). Chose et espace. Leçons de 1907. Paris: P.U.F.

Husserl, E. (1989b). L'arche-originaire Terre ne se meut pas. Recherches fondamentales sur l'origine phénoménologique de la spatialité de la nature. Paris: Minuit.

Husserl, E. (1995). La Tierra no se mueve. Madrid: Facultad de Filosofía, Universidad Complutense.

Husserl, E. (2001). Die 'Bernauer Manuskripte' über das Zeitbewußtsein (1917/18). Netherlands: Rudolf Bernet y Dieter Lohmar, Kluwer Academic Publishers, Dordrecht.

Husserl, E. (2002a). Logische Untersuchungen. Ergänzungsband. Erster Teil. Entwürfe zur Umarbeitung der VI. Untersuchung und zur Vorrede für die Neuauflage der Logischen Untersuchungen (Sommer 1913). Netherlands: Ulrich Melle - Kluwer Academic Publishers - The Hague.

Husserl, E. (2002b). Lecciones de fenomenología de la conciencia interna del tiempo. Madrid: Trotta. 
Husserl, E. (2002c).Zurphänomenologischen Reduktion. Texte aus dem Nachlass (1926-1935). Netherlands: Sebastian Luft - Kluwer Academic Publishers - Dordrecht.

Merleau-Ponty, M. (1960). Le philosophe et son ombre. Paris: Gallimard.

Merleau-Ponty, M. (1968). Husserl aux limites de la phénoménologie. Paris: Gallimard.

Merleau-Ponty, M. (1996). «La philosophie aujourd'hui». En: Notes de cours 1959-1961. Paris: Gallimard.

Merleau-Ponty, M. (1974). «Philosophie et non-philosophie depuis Hegel». En: Notes de cours 1959-1961 (pp. 269-352). Paris: Gallimard.

Merleau-Ponty M. (1996). Notes de cours 1959-1961. Paris: Gallimard.

Merleau-Ponty, M. (1997). Philosophy and Non-Philosophy since Merleau-Ponty. Noethwestern Univ. Press.

Merleau-Ponty, M. (1998). Husserl aux limites de la phénoménologie Paris: P.U.F.

Merleau-Ponty, M. (1999). Le visible et l'invisible. Paris: Gallimard.

Merleau-Ponty, M. (2000). Fenomenología de la Percepción. Barcelona: Península.

Merleau-Ponty, M. (2001). «Non-Philosophie et Philosophie avec deux inédits sur la musique ». En Chiasmi International, (3).

Patoĉka, J. (2004). El movimiento de la existencia humana. Madrid: Ediciones Encuentro.

Saint-Aubert, E. (2006). Vers une ontologie indirecte. Sources et enjeux critiques de l'appel à I'ontologie chez Merleau-Ponty. Paris: Ed. Vrin.

Schopenhauer, A. (2004). "Lección VIII, Sobre el componente subjetivo del placer estético". En: Lecciones sobre metafísica de lo bello. Valencia: Publicaciones de la Universitat de Valencia. 